\title{
Perfluorinated Compounds and Total and Extractable Organic Fluorine in Human Blood Samples from China
}

\begin{abstract}
LEO W. Y. YEUNG, ${ }^{\dagger}$ YUICHI MIYAKE, SACHI TANIYASU, ${ }^{\ddagger}$ YUAN WANG, ${ }^{\dagger}$ HONGXIA YU, ${ }^{\circledR} \mathrm{M}$. K. SO, ${ }^{+}$ GU IB I N J I A G, " YONGNING WU, ${ }^{\perp}$

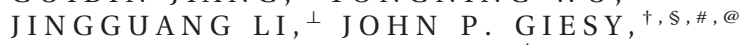
NOBUYOSHI YAMASHITA, ${ }^{*}, \neq$ AND PAUL K. S. LAM*, †

Centre for Coastal Pollution and Conservation, Department of Biology and Chemistry, City University of Hong Kong, Tat Chee Avenue, Kowloon, Hong Kong SAR, People's Republic of China, National Institute of Advanced Industrial Science and Technology, 16-1 Onogawa, Tsukuba, Ibaraki 305-8569, Japan, State Key Laboratory of Pollution Control and Resources, School of the Environment, Nanjing University, Nanjing 210093, People's Republic of China, State Key Laboratory of Environmental Chemistry and Ecotoxicology, Research Center for Eco-Environmental Sciences, Chinese Academy of Sciences P.O. Box 2871, Beijing, 100085, China, Center for Diseases Control and Prevention, 29, Nanwei Road, Beijing, 100050, China, Department of Veterinary Biomedical Sciences and Toxicology Centre, University of Saskatchewan, Saskatoon, Saskatchewan, Canada, and Zoology Department, National Food Safety and Toxicology Center and Center for Integrative Toxicology, Michigan State University, East Lansing, Michigan 48824
\end{abstract}

Received March 3, 2008. Revised manuscript received May 29, 2008. Accepted May 29, 2008.

An improved extraction (ion pairing) and cleanup (ENVI-carb and solid phase extraction) method was developed for analysis of perfluorinated compounds (PFCs) in human whole blood samples from China. Ten PFCs including PFOS, PFHxS, PFOSA, PFDoDA, PFUnDA, PFDA, PFNA, PFOA, PFHpA, and PFHxA were detected in the blood samples $(n=30)$ from five cities (Jintan, Nanjing, Guiyang, Beijing, and Shenyang). PFOS was found to be the dominant PFC ranging from $0.446-83.1 \mathrm{ng} /$ $\mathrm{mL}$. Total fluorine (TF) and extractable organic fluorine (EOF) also were measured in the blood samples using combustion ion chromatography for fluorine. Analysis of known PFCs and extractable organic fluorine showed that known PFCs could account for $>70 \%$ of EOF in samples from Beijing, Shenyang, and Guiyang, whereas known PFCs could only account for $\sim 30 \%$ of EOF in samples from Jintan. Results of the present study indicated the presence of substantial amounts of unidentified

* Address correspondence to either author. (N.Y.) Tel.: +81-29861-8335; fax: +81-29-861-8335; e-mail: nob.yamashita@aist.go.jp. (P.K.S.L.) Tel.: +852-2788-7681; fax: +852-2788-7406; e-mail: bhpksl@cityu.edu.hk.

${ }^{\dagger}$ City University of Hong Kong.

${ }^{\ddagger}$ National Institute of Advanced Industrial Science and Technology.

${ }^{\S}$ Nanjing University.

"Chinese Academy of Sciences.

${ }^{\perp}$ Center for Diseases Control and Prevention.

\# University of Saskatchewan.

${ }^{\circledR}$ Michigan State University. organic fluorine in human blood samples from Jintan.

Characterization and identification of these unidentified

fluorinated compounds will be instructive.

\section{Introduction}

The ubiquitous occurrence of perfluorsinated compounds (PFCs) in environmental samples has drawn much attention (1-5). Perfluorooctane sulfonate (PFOS) is commonly detected in environmental samples, but both PFOS and perfluorooctanoate (PFOA), another important PFC due to its wide use and application, are of great concern because of their global occurrence in human blood and serum samples (3, 6-9). Although toxicological studies have demonstrated that adverse health effects such as reduced body weight, hepatocellular hypertrophy, and decreased serum cholesterol and triglycerides occurred in rats and monkeys after PFOS exposure $(10,11)$, and genes involved in the peroxisomal and mitochondrial beta-oxidation pathways were affected in rats exposed to PFOA (12), the measured PFC concentrations in blood samples from general human populations (6) were much lower than those measured in these test animals (PFOS No observable adverse effect level (NOAEL): $44 \mu \mathrm{g} /$ $\mathrm{mL}$ for male rats and $64 \mu \mathrm{g} / \mathrm{mL}$ for female rats; PFOS NOAEL for male monkeys was $82.6 \mu \mathrm{g} / \mathrm{mL}$ and was $66.8 \mu \mathrm{g} / \mathrm{mL}$ for female monkeys) $(10,11)$. Elevated PFOS $(1.32 \mu \mathrm{g} / \mathrm{mL})$ and PFOA $(1.78 \mu \mathrm{g} / \mathrm{mL})$ concentrations were measured in perfluorooctanesulfonyl fluoride manufacturing workers (13). Total bilirubin $(0.008 \mathrm{mg} / \mathrm{dL}$ decline $/ \mu \mathrm{g} / \mathrm{mL})$ and serum aspartate aminotransferase ( 0.35 unit increase $/ \mu \mathrm{g} / \mathrm{mL})$ were related to serum PFOA concentrations but not other liver enzymes (14). There were no significant positive correlations between serum PFOA concentrations and measured health parameters such as thyroid-stimulating hormones or red cell indices (15). In addition, a recent study suggested that there was little support for an association between bladder cancer and PFOS exposure for perfluorooctanesulfonyl fluoride manufacturing workers (16). Information concerning the health effects of PFCs at the reported exposure level of the general public is inconclusive.

The U.S. Interagency Testing Committee (ITC) identified 50 PFCs of interest because of their potential for environmental persistence and long-range transport (www.epa.gov/ opptintr/itc). Measuring PFCs in environmental samples allows for a gross understanding of the degree of PFC pollution in the environment; however, this understanding depends on the efficacy and efficiency of existing extraction methods and the availability of authentic analytical standards. PFOS and PFOA are the dominant PFCs in environmental samples, but it is also known that these compounds only account for a portion of the PFCs that are present in environmental and biological matrices $(17,18)$. Other fluorochemicals, such as fluorotelomer alcohols and shorter and longer chain perfluorocarboxylates (PFCAs), have become important elements in monitoring surveys and risk evaluations. Currently, more than 18 PFCs can be detected in seawater, animal tissues, and human samples with the help of advanced analytical instrumentation and analytical standards $(19,20)$. Even though analytical methods exist to measure 41 different PFCs (21), it is technically challenging, if not impossible, to determine all of the individual PFCs in a sample.

Total organohalogens have been measured in a variety of environmental samples (22-24). Most of these studies measured total extractable chlorine $(\mathrm{EOCl})$, bromine $(\mathrm{EOBr})$, and iodine (EOI), and there is a paucity of data regarding 
extractable fluorine (EOF) (18) due to analytical limitations. Refined combustion ion chromatography for fluorine (CICF) and improvements in analytical extraction methods for the separation of organic and inorganic fluorine were developed recently $(17,18)$. This improved method is applicable to trace fluorine analyses at parts per trillion levels in water (17) and parts per billion levels in blood samples (18). The concentration of total fluorine (TF) in any sample is equal to the sum of inorganic fluorine (IF) and organic fluorine (OF) concentrations in that sample. TF was determined in blood samples (18), but OF could not be measured accurately because the analytical result depended on the extraction method used. Therefore, only a proportion of the $\mathrm{OF}$ in a sample could be extracted (i.e., extractable organic fluorine (EOF), while the OF that could not be extracted using the developed extraction methods was regarded as nonextractable organic fluorine (NEOF) (Supporting Information Figure 1)). In the present study, the amount of TF in the blood samples was measured without any fractionation. Two fractionation steps using ion-pairing extraction (EOF Fr1) and hexane extraction (EOF Fr2) were used for the determination of the amount of extractable organic fluorine in the sample (Supporting Information Figure 2).

The application of these new extraction and analytical methods showed that known PFCs accounted for $>80 \%$ of the EOF fraction in human blood samples from Japan and the U.S., while IF and NEOF accounted for $>70 \%$ of the TF in those samples (18). Measurement of TF, EOF, and IF allows for a better understanding of the degree of PFC contamination and can help to identify the presence of other unidentified fluorochemicals and sources of contamination that are introduced into the environment.

The results of investigations of PFC levels in human blood in China have been reported elsewhere $(9,25,26)$. This is the first report concerning $\mathrm{TF}$ in human blood samples from China. The degree of OF contamination in the Chinese population is still unknown. In view of this, blood samples from four cities from the previous study were selected for TF analyses (9). Samples from Shenyang and Jintan were selected because those samples contained the greatest and lowest PFC concentrations, respectively; the samples from Guiyang and Beijing had similar PFC composition profiles and were selected for TF analysis to investigate the patterns of known PFCs and OF in these samples. Samples from Nanjing, a new location, also were analyzed to provide further information on PFC concentrations in China.

The challenge for quality in the determination of PFC concentrations in environmental and human samples has been discussed recently (27). Data uncertainties result from both the lack of analytical standards and the matrix effects from ionization problems during mass spectrometric detection and quantification of PFCs. Ion-pairing extraction methods (28) and formic acid digestion with solid phase extraction (SPE) (29) were the common methods for the extraction of PFCs from human blood samples, while ENVIcarb (graphitized carbon) (30) was used as a cleanup method. In the present study, we applied cleanup processes using ENVI-carb, followed by SPE after the ion-pairing extraction, to lower the background interferences for some PFCs such as perfluoroheptanoic acid ( $\mathrm{PFHpA}$ ) and perfluorononanoic acid (PFNA).

The objectives of the present study were (i) to determine TF, EOF, and known PFCs in human blood samples and (ii) to evaluate the relative proportions of known PFCs to organic and total fluorine in human blood samples to investigate the presence of other unidentified organic fluorinated compounds. Improved cleanup methods using ENVI-carb and SPE were described and applied to the blood samples.

\section{Materials and Methods}

Details of chemicals and reagents used and experimental procedures employed in the present study are given in the Supporting Information.

Sample Collection. Samples of whole human blood from Jintan, Nanjing, Shenyang, Guiyang, and Beijing were collected from volunteer donors from local universities or hospitals in China in 2004 following the same protocol as described in the previous study (9). Detailed sample information is shown in Supporting Information Table 1. The whole blood samples were stored in polypropylene (PP) containers or vacutainers at $-20{ }^{\circ} \mathrm{C}$ until analysis.

Individual PFC Analysis. Thirteen individual PFCs were analyzed using the ion-pairing method, the details of which are described elsewhere (28) and are summarized in Supporting Information Figures 1 and 2. To reduce interference, further ENVI-carb (30) and SPE (19) cleanup were applied to the extract (Supporting Information Figure 2).

TF, IF, and EOF Analyses. An aliquot of each blood sample was subjected to fractionation (Supporting Information Figure 2) for the analysis of TF, IF, and EOF, using extraction procedures described elsewhere (18). TF was determined by taking $0.1 \mathrm{~mL}$ of whole blood on a silica boat and placing it directly into the CIC. EOF (Frl) was quantified from the extract of ion pairing (MTBE fraction), and EOF (Fr2) was quantified after another extraction of the ion pairing with hexane using CIC. Details of the procedure are provided in the Supporting Information.

Instrumental Analysis and Quantification. Concentrations of PFCs in whole blood samples were analyzed by the use of high-performance liquid chromatography with tandem mass spectrometry (HPLC-MS/MS). Separation of analytes was performed using an Agilent HP1100 liquid chromatograph (Agilent, Palo Alto, CA) interfaced with a Micromass Quatro Ultima Pt mass spectrometer (Waters Corp., Milford, MA) operated in electrospray negative mode. The details of separation using HLPC, desolvation gas flow, temperature, collision energies, cone voltages, and MS/MS parameters for the instrument were optimized for individual analytes and are reported elsewhere (ref 19, and Supporting Information). In this study, the external calibration curve was used for quantification, the details of which can be found in the Supporting Information. Cleanup methods were applied to obtain an extract that was similar to the standards in the solvent. This method has given reliable results as indicated by the international interlaboratory calibration exercise (31).

TF and EOF were determined using modified CIC, by the combination of an automated combustion unit (AQF-100 type AIST; Dia Instruments Co., Ltd.) and an ion chromatography system (ICS-3000 type AIST; Dionex Corp., Sunnyvale, CA). The details for analyses are given elsewhere $(17,18)$ and in the Supporting Information.

Quality Assurance/Quality Control. Individual PFC Analyses. To achieve lower detection limits for HPLC-MS/MS, all accessible polytetrafluoroethylene (PTFE) and other fluoropolymer materials were removed from the instruments and apparatus to minimize the background signal due to contamination. PP tubes and septa were selected after a thorough checking of blanks. Impurities of each standard chemical were tested at the nanogram per milliliter level, and no contamination from chemical reagents was observed. Procedural blanks associated with every 10 samples were tested to check for possible laboratory contamination and interferences. The blank levels in the tube used for sample collection were checked and found to be $<7 \mathrm{pg} / \mathrm{mL}$ in washed methanol for all analytes. All target chemicals were spiked into test samples of blood (for matrix-spike recovery tests), and the samples were extracted and analyzed following the same procedures as described previously (Supporting In- 
formation Table 2). Recoveries were evaluated by subtracting background levels from detected concentrations. PFC concentrations were not corrected for recoveries.

TFAnalyses. The gases used for CIC are a source of fluorine contamination. Therefore, high-purity gases were used (Ar: 99.9999\% and $\mathrm{O}_{2}$ : 99.9995\%). The ion chromatograph tubing, gas lines, valves, and regulator, which contained materials or parts made of PTFE, were replaced with stainless steel, polyetheretherketone, or polyethylene tubing. Furthermore, a gas purifier containing activated carbon was placed in the gas line to remove trace levels of fluorine from the gases. Analyses of total and extractable fluorine were conducted in duplicate. Analysis of PFOS standard 10 and $100 \mathrm{ng} / \mathrm{mL}$ in $\mathrm{MeOH}$ showed 95\% (SD: 5\%) and 98\% (SD: 3\%) recovery, respectively. In-house reference material (pig's blood) was analyzed before and after every $10 \mathrm{CIC}$ injections (TF mean: $173 \mathrm{ng} \mathrm{F} / \mathrm{mL}$ and SD: $13 \mathrm{ng} / \mathrm{mL}, n=10$ ) to check the overall efficiency of the combustion process.

Statistical Analyses. A nonparametric Kruskal-Wallis test was used to assess the differences in concentrations of EOF measured in the blood samples among locations. A nonparametric Mann-Whitney test was used to assess the differences in concentrations of EOF measured in the blood samples between genders. A Spearman rank correlation was used to examine possible correlations between EOF and TF with age in the blood samples. A significant level was accepted at $\alpha=0.05$.

\section{Results and Discussion}

Procedure blank and recovery tests were conducted on each batch of samples and are summarized in Supporting Information Table 2. The procedural blanks, recoveries, and matrix-spike recoveries, using the same blood matrices and spiking level (1 ng of each PFC), were acceptable and comparable to those from a previous report (Supporting Information Table 2). The modified ion-pairing extractions (i.e., with further cleanup methods), PFBS, PFHpA, 8:2FTCA, and 8:2FTUCA, now could be determined using the present method. In general, PFOS, PFHxS, PFOSA, PFDoDA, PFUnDA, PFDA, PFNA, PFOA, PFHpA, and PFHxA were detected in the samples, whereas PFBS, 8:2 FTUCA, and 8:2 FTCA concentrations were all below LOQs (Table 1).

The PFC concentrations in human blood from individuals living in different Chinese cities are summarized in Table 1. The results from the present study agreed with those from the previous study (9) in that individuals from Shenyang had the greatest PFOS and PFOSA concentrations (56.3 and $1.65 \mathrm{ng} /$ $\mathrm{mL}$, respectively). However, PFOS and PFOSA concentrations in individuals living in Nanjing (1.4 and $0.112 \mathrm{ng} / \mathrm{mL}$, respectively) were lower than those in blood from Jintan, which contained the lowest concentrations in the last report (9). Among the perfluorocarboxylates (PFCAs) measured, PFNA could be detected in all blood samples and contributed the major proportion of total PFCAs, followed by PFUnDA, which also frequently was detected in the blood samples with the exception of Jintan. PFHpA and PFHxA could only be detected in a few samples from the present study. The greatest PFCA concentrations were found in blood samples of individuals living in Jintan, whereas concentrations in individuals living in the other four cities were 7 times lower.

The PFC composition profiles of human blood samples varied among locations (Supporting Information Figure 3), but PFOS was always the dominant PFC, contributing $\sim 60-90 \%$ of the total PFCs. The composition profiles among Shenyang, Guiyang, and Beijing were similar, but composition profiles from Jintan and Nanjing showed that PFCAs comprised a greater proportion of total PFCs in these samples. These results suggested different sources of contamination and/or different routes of exposure in Jintan and Nanjing as compared to the other cities. 
TABLE 2. TF, EOF, and Known PFC Concentrations (ng F/mL) in Chinese Blood Samples ${ }^{a}$

sum known PFCs (ng F/mL)

$\begin{array}{lll} & \text { LOQ } & \text { N/A } \\ \text { Shenyang } & \text { mean } & 37.2 \\ n=5 & \text { SD } & 12.3 \\ \text { Beijing } & \text { mean } & 10.1 \\ n=5 & \text { SD } & 4.41 \\ \text { Guiyang } & \text { mean } & 15.0 \\ n=5 & \text { SD } & 10.1 \\ \text { Jintan } & \text { mean } & 6.02 \\ n=5 & \text { SD } & 2.53 \\ \text { Nanjing } & \text { mean } & 1.32 \\ n=10 & \text { SD } & 0.608\end{array}$

EOF (Fr1)-MTBE (ng F/mL)

6

43.4

13.2

17.4

6.73

17.0

8.48

24.5

11.1

$<6$
EOF (Fr2)-hexane (ng F/mL)

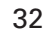

$<32$

$<32$

$<32$

$<32$

$<32$
TF (ng F/mL)

6

166

53.2

117

55.1

102

29.6

60.6

19.5

77.0

23.1

${ }^{a}$ N/A: not applicable.

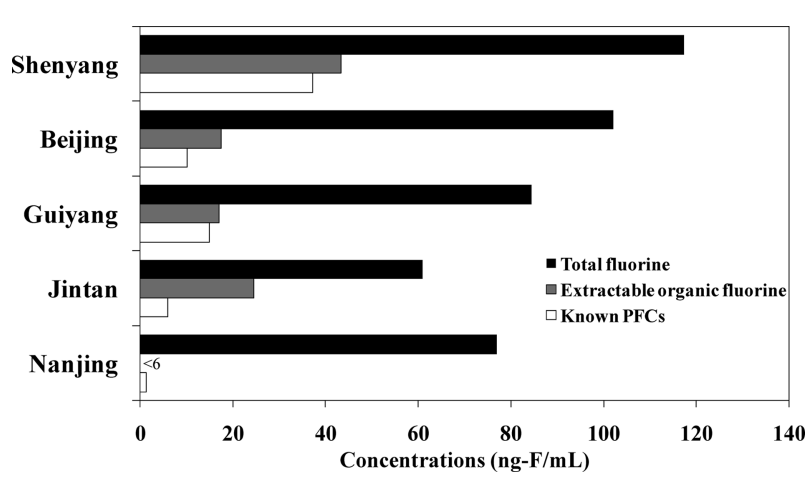

FIGURE 1. TF, EOF, and known PFC concentrations (ng F/mL).

The occurrence of OFs was reported as early as the 1960s in serum samples from fluorochemical plant workers (32), and the conventional PFC monitoring studies could only report some of the known PFCs in the environmental samples due to analytical limitations. It is important to know how much OF is present in human blood and how much of OF is accounted for by PFCs. In the present study, further information on fluorine contamination was obtained using a refined and improved CIC-F method (Supporting Information Figure 1), in which EOF was measured as both MTBEextractable fluorine (Frl) and hexane-extractable fluorine fractions (Fr2). EOF (Fr1) could be measured in samples from Beijing, Guiyang, Jintan, and Shenyang, whereas samples from Nanjing were all below the limit of detection $(<6 \mathrm{ng}$ $\mathrm{F} / \mathrm{mL}$ ) (Table 2 and Figure 1). Shenyang had the greatest EOF (Fr1) concentrations, whereas Jintan, Beijing, and Guiyang had lower but similar EOF (Fr1) concentrations. EOF concentrations among these cities were not significantly different $[\mathrm{H}(3, n=20)=3.11$ and $P=0.213]$. No age-related $\left(R^{2}=0.0429, n=20\right.$, and $\left.P=0.315\right)$ or gender-related $(U=$ $31, n=20$, and $P=0.630$ ) accumulation was observed. These observations corresponded to previous studies that reported no related accumulation of PFCAs such as PFOA and PFNA in human blood samples $(3,9)$.

EOF varied with the method used for extraction, and therefore, EOF levels could only be compared when samples were extracted using the same method. The analytical procedures used in the present study were the same as those of a previous study (18), allowing the results from the present study to be compared with the previous study. EOF (Fr1) concentrations in blood samples from Japanese individuals ranged from $<6$ to $8.89 \mathrm{ng} \mathrm{F} / \mathrm{mL}$, whereas those in individuals from the U.S. ranged from 17.8 to $59 \mathrm{ng} \mathrm{F} / \mathrm{mL}$. Samples from China were within the range of Japan and the U.S. $(<6-60$ $\mathrm{ng} \mathrm{F} / \mathrm{mL}$ ). As PFCs were in the subcategory of EOF (Fr1), it is interesting and important to know how much EOF (Fr1) can be explained by PFCs (Figure 2). Known PFCs contributed the major proportion of EOF (Fr1) in samples from Guiyang

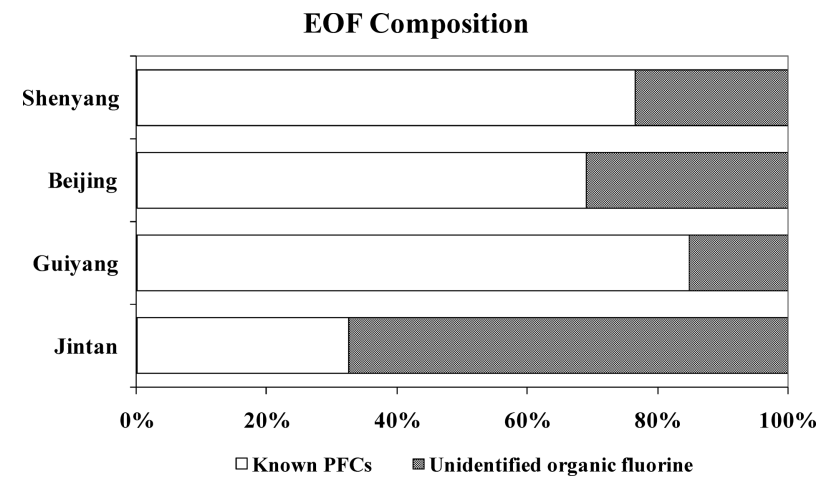

FIGURE 2. Composition profile of EOF in Chinese human blood.

(85\%), Beijing (57\%), and Shenyang (77\%); these large known PFC contributions to EOF were similar to another study that showed that PFCs accounted for a major portion of EOF in samples from the U.S. (85-103\%) and Japan (84\%) (18). In contrast, known PFCs only accounted for $33 \%$ of the EOF in samples from Jintan. These results convey three important messages: first, known PFCs were the major OF contaminants (>60\%) in human blood from individuals living in Guiyang, Beijing, and Shenyang; second, there remained a large proportion of unidentified fluorinated chemicals in blood samples from individuals living in Jintan; and third, Jintan is a small city that differs from Beijing and Shenyang in terms of being much less industrially developed. There may be different exposure pathways or contamination sources of organic fluorinated compounds for individuals living in Jintan as compared to the other cities.

EOF (Fr1) probably consisted of polar fluorinated compounds that could be extracted using ion-pairing reagents (i.e., TBA and MTBE). These compounds might include unknown PFCs (C6-C12 PFCAs and C4, C6, and C8 PFASs). However, some short-chain PFCAs such as C2 and C3 and long-chain PFCAs such as $\mathrm{C} 14, \mathrm{C} 16$, and $\mathrm{C} 18$ could be present in the samples but were not all extracted and measured accurately. Other known fluorochemicals such as $N$-ethyl perfluorooctanesulfonamidoacetate (PFOSAA), N-methyl perfluorooctanesulfonamidoacetate (M570), and perfluorooctanesulfonamidoacetate (M556) were detected in human blood samples $(3,33)$ but were not measured in the present study. Furthermore, fluorinated chemicals have been widely used in different applications as anesthetics, pesticides, fungicides, and so on (reviewed in ref 34). All of these fluorochemicals might account for parts of unidentified fluorinated chemicals in the blood samples. However, the identification of these unidentified fluorines was separate from the scope of the present study, and further investigation of these fluorinated chemicals is needed.

EOF (Fr2) consisted of semi- or nonvolatile fluorinated compounds that were extracted by hexane. In the present 
Fluorine composition

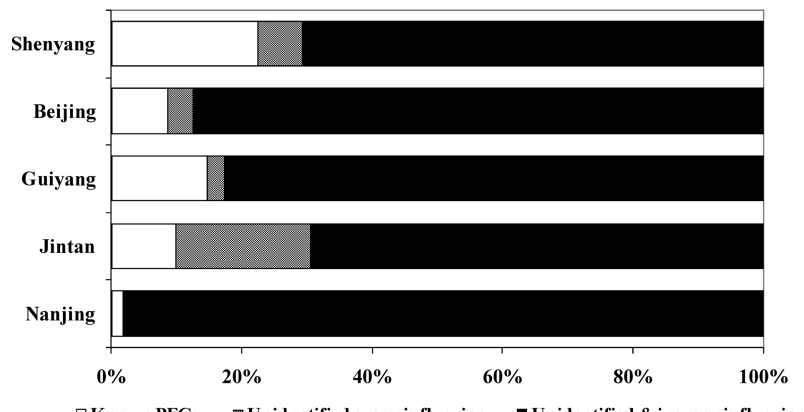

$\square$ Known PFCs $\quad$ Unidentified organic fluorine Unidentified \& inorganic fluorine

FIGURE 3. Composition profile of fluorine in Chinese human blood.

study, $>90 \%$ (28 of 30 samples) of the blood samples was below the LOQ $(<32 \mathrm{ng} / \mathrm{mL})$. Because of a relatively higher detection limit for EOF (Fr2), it was difficult to ascertain as to which fractions, EOF (Fr1) or EOF (Fr2), contributed more to the organofluorine contamination. However, EOF (Fr1) of the samples from Shenyang was greater than the detection limit for EOF (Fr2), and so it is conceivable that organofluorine contamination may be mainly attributed to EOF (Fr1) (or PFCs because they accounted for $\sim 90 \%$ of this fraction).

TF concentrations in Chinese blood ranged from 60.6 to 166 ng F/mL (Table 2 and Figure 1). TF in Chinese blood samples has not been reported to date, and therefore, comparisons with other studies on the Chinese population could not be made. The greatest mean TF concentrations were found in samples from Shenyang and the least mean concentrations occurred in samples from Nanjing (Table 2 and Figure 1). The TF concentrations in the Chinese population were comparable to those in samples from Japan (181-262 ng F/mL) and the U.S. (140-189 ng F/mL) (18). Comprehensive mass balance analysis of fluorine in Chinese blood samples showed that NEOF and IF accounted for a major proportion of TF and that known PFCs contributed $2-30 \%$ of TF in these samples (Figure 3 ). These observations were similar to a previous study (18) that showed that known PFCs contributed $<30 \%$ of TF, with a large proportion of fluorine belonging to IF and NEOF in those blood samples.

As it is unlikely or perhaps impossible to identify or measure all PFCs or unidentified OF, analyses of TF, particularly of EOF, could provide important insights into environmental risk assessment. The results of TF analyses of samples from Shenyang and Jintan suggest at least two possible courses of action. In the first approach, if it is assumed that EOF (Fr1) is composed of all the PFCs, then known PFCs accounted for the major proportion of EOF in samples from Shenyang, indicating that effort should be directed toward indentifying major contamination sources of these known PFCs and that proper management should be implemented to reduce these sources of contamination in this city. If the proportion of EOF that is unknown in the Jintan samples is assumed to be made up solely of unidentified PFCs, the analyses would indicate that these unknown PFCs were being used or released in the region and that the exposure pathways might be different from those in Shenyang. In terms of allocation of resources toward environmental assessment and management, these results suggest that more attention should be given to Shenyang, whereas Jintan might be a lower priority for environmental management, if the decision was made based only on contamination by known PFCs. In the second approach, if the unknown EOF (Frl) is assumed to be fluorinated compounds other than PFCs, then people living in Jintan are exposed to other kinds of OF that might have similar physical properties and similar exposure pathways as PFCs. Effort should therefore be focused on the identification of these unidentified OFs instead of on PFCs (e.g., using other analytical techniques such as NMR or by measuring other fluorinated chemicals such as fluorinated pharmaceuticals or pesticides).

\section{Acknowledgments}

The work described in this paper was supported by a grant from the Research Grants Council of the Hong Kong Special Administrative Region, China (CityU160507). This work was undertaken during the tenure of a City University Postgraduate Studentship to L.W.Y.Y. We gratefully acknowledge the donors who voluntarily contributed blood samples for this study. We thank Dr. Margaret Murphy (City University of Hong Kong) for critical suggestions of this manuscript.

\section{Supporting Information Available}

Details of chemicals and reagents used and experimental procedures employed in the present study. Table 1: details of human blood samples analyzed in 2004; Table 2: procedural blanks, recoveries, and matrix-spiked recoveries for PFCs; Figure 1: components of total fluorine analyses; Figure 2: schematic diagram showing extraction and analysis methods for individual PFCs, EOF, and TF; and Figure 3: PFC composition profiles in Chinese blood samples. This material is available free of charge via the Internet at http:// pubs.acs.org.

\section{Literature Cited}

(1) Kannan, K.; Koistinen, J.; Beckmen, K.; Evans, T.; Gorzelany, J. F.; Hansen, K. J.; Jones, P. D.; Helle, H.; Nyman, M.; Giesy, J. P. Accumulation of perfluorooctane sulfonate in marine mammals. Environ. Sci. Technol. 2001, 35, 1593-1598.

(2) Kannan, K.; Corsolini, S.; Falandysz, J.; Oehme, G.; Focardi, S.; Giesy, J. P. Perfluorooctanesulfonate and related fluorinated hydrocarbons in marine mammals, fish, and birds from coasts of the Baltic and Mediterranean Seas. Environ. Sci. Technol. 2002, 36, 3210-3216.

(3) Olsen, G. W.; Church, T. R.; Miller, J. P.; Burris, J. M.; Hansen, K. L.; Lundberg, J. K.; Armitage, J. B.; Herron, R. M.; Mebhidizadehkashi, Z.; Nobiletti, J. B.; O’Neill, E. M.; Mandel, J. H.; Zobel, L. R. Perfluorooctanesulfonate and other fluorochemicals in the serum of American Red Cross adult blood donors. Environ. Health Perspect. 2003, 111, 1892-1901.

(4) Giesy, J. P.; Kannan, K. Global distribution of perfluorooctane sulfonate in wildlife. Environ. Sci. Technol. 2001, 35, 1339-1342.

(5) Giesy, J. P.; Kannan, K. Perfluorochemical surfactants in the environment. Environ. Sci. Technol. 2002, 36, 146-152.

(6) Kannan, K.; Corsolini, S.; Falandysz, J.; Fillmann, G.; Kumar, K. S.; Loganathan, B. G.; Mohd, M. A.; Olivero, J.; Van Wouwe, N.; Yang, J. H.; Aldous, K. M. Perfluorooctane sulfonate and related fluorochemicals in human blood from several countries. Environ. Sci. Technol. 2004, 38, 4489-4495.

(7) Guruge, K. S.; Taniyasu, S.; Yamashita, N.; Wijeratna, S.; Mohotti, K. M.; Seneviratne, H. R.; Kannan, K.; Yamanaka, N.; Miyazaki, S. Perfluorinated organic compounds in human blood serum and seminal plasma: A study of urban and rural tea worker populations in Sri Lanka. J. Environ. Monit. 2005, 7, 371-377.

(8) Karrman, A.; Muleller, J. F.; van Bavel, B.; Harden, F.; Toms, L. L.; Lindstrom, G. Levels of 12 perfluorinated chemicals in pooled Australian serum, collected 2002-2003, in relation to age, gender, and region. Environ. Sci. Technol. 2006, 40, 37423748.

(9) Yeung, L. W.; So, M. K.; Jiang, G.; Taniyasu, S.; Yamashita, N.; Song, M.; Wu, Y.; Li, J.; Giesy, J. P.; Guruge, K. S.; Lam, P. K. S. Perfluorooctanesulfonate and related fluorochemicals in human blood samples from China. Environ. Sci. Technol. 2006, 40, 715720.

(10) Seacat, A. M.; Thomford, P. J.; Hansen, K. J.; Clemen, L. A.; Eldridge, S. R.; Elcombe, C. R.; Butenhoff, J. L. Sub-chronic dietary toxicity of potassium perfluorooctane sulfonate in rats. Toxicology 2003, 183, 117-131.

(11) Seacat, A. M.; Thomford, P. J.; Hansen, K. J.; Olsen, G. W.; Case, M. T.; Butenhoff, J. L. Subchronic toxicity studies on perfluorooctanesulfonate potassium salt in cynomolgus monkeys. Toxicol. Sci. 2002, 68, 249-264.

(12) Guruge, K. S.; Yeung, L. W. Y.; Yamanaka, N.; Miyazaki, S.; Lam, P. K. S.; Giesy, J. P.; Jones, P. D.; Yamashita, N. Gene expression profiles in rat liver treated with perfluorooctanoic acid (PFOA). 
Toxicol. Sci. 2006, 89, 93-107.

(13) Olesn, G. W.; Burris, J. M.; Burlew, M. M.; Mandel, J. H. Epidemiologic assessment o worker serum perfluorooctanesulphonate (PFOS) and perfluorooctanoate (PFOA) concentrations and medical surveillance examinations. J. Occup. Environ. Med. 2003, 45, 260-270.

(14) Sakr, C. J.; Leonard, R. C.; Kreckmann, K. H.; Slade, M. D.; Culler, M. R. Longitudinal study of serum lipids and liver enzymes in workers: Occupational exposure to ammonium perfluorooctanoate. J. Occup. Environ. Med. 2007, 49, 872-879.

(15) Emmett, E. A.; Zhang, H.; Shofer, F. S.; Freeman, D.; Rodway, N. V.; Desai, C.; Shaw, L. M. Community exposure to perfluorooctanoate: Relationships between serum levels and certain health parameters. J. Occup. Environ. Med. 2006, 48, 771-779.

(16) Alexander, B. H.; Olsen, G. W. Bladder cancer in perfluorooctanesulfonyl fluoride manufacturing workers. Ann. Epidemiol. 2007, 17, 471-478.

(17) Miyake, Y.; Yamashita, N.; Rostkowski, P.; So, M. K.; Taniyasu, S.; Lam, P. K. S.; Kannan, K. Determination of trace levels of total fluorine in water using combustion ion chromatography for fluorine: A mass balance approach to determine individual perfluorinated chemicals in water. J. Chromatogr., A 2007, 1143, 98-104.

(18) Miyake, Y.; Yamashita, N.; So, M. K.; Rostkowski, P.; Taniyasu, S.; Lam, P. K. S.; Kannan, K. Trace analysis of total fluorine in human blood using combustion ion chromatography for fluorine: A mass balance approach for the determination of known and unknown organofluorine compounds. J. Chromatogr., A 2007, 1154, 214-221.

(19) Taniyasu, S.; Kannan, K.; So, M. K.; Gulkowska, A.; Sinclair, E.; Okazawa, T.; Yamashita, N. Analysis of fluorotelomer alcohols, fluorotelomer acids, and short- and long-chain perfluorinated acids in water and biota. J. Chromatogr., A 2005, 1093, 89-97.

(20) Tao, L.; Kannan, K.; Kajiwara, N.; Costa, M. M.; Fillmann, G.; Takahashi, S.; Tanabe, S. Perfluorooctanesulfonate and related fluorochemicals in albatrosses, elephant seals, penguins, and polar skuas from the Southern Ocean. Environ. Sci. Technol. 2006, 40, 7642-7647.

(21) Ahrens, L.; Pla $\beta$ mann, M.; Temme, C.; Ebinghaus, R. Determination of per- and polyfluorinated alkyl compounds using liquid chromatography tandem mass spectrometry in water samples. Organohalogen Compd. 2007, 69, 2804-2807.

(22) Kawano, M.; Falandysz, J.; Wakimoto, T. Instrumental neutron activation analysis of extractable organohalogens in the Antarctic Weddell seal (Leptonychotes weddelli). J. Radioanal. Nucl. Chem. 2007, 272, 501-504.

(23) Xu, D.; Deng, L.; Chai, Z.; Mao, X. Organohalogenated compounds in pine needles from Beijing City, China. Chemosphere
2004, 57, 1343-1353.

(24) Matsuaki, M.; Kashima, Y.; Kawano, M.; Mastuda, M.; Ambe, K.; Wakimoto, T.; Doi, T. Dioxin-like potencies and extractable organohalogens (EOX) in medical, municipal, and domestic waste incinerator ashes in Japan. Chemosphere 2003, 53, 971980.

(25) Jin, Y.; Liu, X.; Zhang, X.; Li, T.; Zhang, Y.; Saito, N.; Sasaki, K.; Koizumi, A. Perfluorooctanesulfonate situation in serum of the common population. Chin. J. Public Health 2003, 19, 12001201.

(26) Jin, Y.; Saito, N.; Harada, K.; Inoue, K.; Koizumi, A. Historical trends in human serum levels of perfluorooctanoate and perfluorosulfonate in Shenyang, China. Tohoku J. Exp. Med. 2007, 212, 63-70.

(27) Van Leeuwen, S. P. J.; Karrman, A.; van Bavel, B.; de Boer, J.; Lindstrom, G. Struggle for quality in determination of perfluorinated contaminants in environmental and human samples. Environ. Sci. Technol. 2006, 40, 7854-7860.

(28) Hansen, K. J.; Clemen, L. A.; Ellefson, M. E.; Johnson, H. O. Compound-specific, quantitative characterization of organic fluorochemicals in biological matrices. Environ. Sci. Technol. 2001, 35, 766-770.

(29) Karrman, A.; van Bavel, B.; Jarnberg, U.; Hardell, L.; Lindstrom, G. Development of a solid-phase extraction-HPLC/single quadrupole MS method for quantification of perfluorochemicals in whole blood. Anal. Chem. 2005, 77, 864-870.

(30) Powley, C. R.; George, S. W.; Ryan, T. W.; Buck, R. C. Matrix effect-free analytical methods for determination of perfluorinated carboxylic acids in environmental matrixes. Anal. Chem. 2005, 77, 6353-6358.

(31) Lindstrom, G.; Karrman, A.; Zammitt, A.; Van Bavel, B.; Van der Veen, I.; Kwadijk, C.; de Boer, J.; van Leeuwen, S. The first worldwide interlaboratory study on perfluorinated compounds in environmental and human samples. Proceedings of the 2005 International Fluoros Symposium: An International Symposium on Fluorinated Alkyl Organics in the Environment, Toronto, Canada, August 18-20, 2005; Mabury, S., Ed.; University of Toronto: Toronto, Canada; p ANA040.

(32) Taves, D. Evidence that there are two forms of fluorine in human serum. Nature (London, U.K.) 1968, 217, 1050-1051.

(33) Olsen, G. W.; Church, T. R.; Larson, E. B.; van Belle, G.; Lundberg, J. K.; Hansen, K. J.; Burris, J. M.; Mandel, J. H.; Zobel, L. R. Chemosphere 2004, 54, 1599-1611.

(34) Key, B. D.; Howell, R. D.; Criddle, C. S. Fluorinated organics in the biosphere. Environ. Sci. Technol. 1997, 31, 2445-2454.

ES800631N 